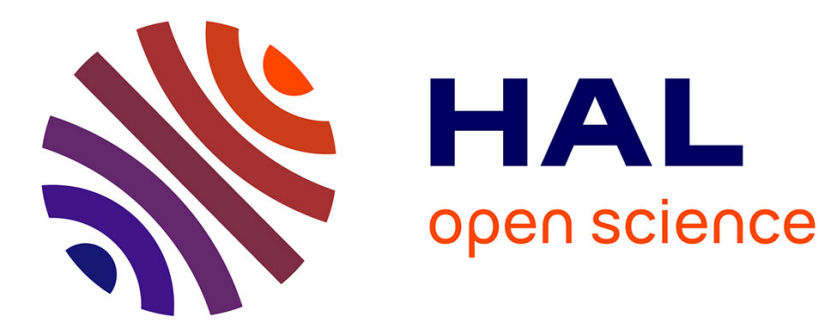

\title{
Synthesis on a class of algebraic differentiators and application to nonlinear observation
}

\author{
Da-Yan Liu, Olivier Gibaru, Wilfrid Perruquetti
}

\section{To cite this version:}

Da-Yan Liu, Olivier Gibaru, Wilfrid Perruquetti. Synthesis on a class of algebraic differentiators and application to nonlinear observation. The 33rd Chinese Control Conference (CCC), Jul 2014, Nanjing,

China. hal-00986345

\section{HAL Id: hal-00986345 \\ https://inria.hal.science/hal-00986345}

Submitted on 2 May 2014

HAL is a multi-disciplinary open access archive for the deposit and dissemination of scientific research documents, whether they are published or not. The documents may come from teaching and research institutions in France or abroad, or from public or private research centers.
L'archive ouverte pluridisciplinaire HAL, est destinée au dépôt et à la diffusion de documents scientifiques de niveau recherche, publiés ou non, émanant des établissements d'enseignement et de recherche français ou étrangers, des laboratoires publics ou privés. 


\title{
Synthesis on a class of algebraic differentiators and application to nonlinear observation
}

\author{
LIU Da-Yan ${ }^{1}$, GIBARU Olivier ${ }^{2,4}$, PERRUQUETTI Wilfrid ${ }^{3,4}$ \\ 1. INSA Centre Val de Loire, Université d'Orléans, PRISME EA 4229, Bourges Cedex 18020, France \\ E-mail: dayan.liu@insa-cvl.fr \\ 2. LSIS (CNRS, UMR 7296), Arts et Métiers ParisTech, Lille Cedex 59046, France \\ E-mail: olivier.gibaru@ensam.eu \\ 3. LAGIS (CNRS, UMR 8219), Ecole Centrale de Lille, Villeneuve d'Ascq 59650, France \\ E-mail: wilfrid.perruquetti@inria.fr \\ 4. Equipe Projet Non-A, INRIA Lille-Nord Europe, Villeneuve d'Ascq 59650, France
}

\begin{abstract}
The recent algebraic parametric method proposed by Fliess and Sira-Ramírez $[1,2]$ has been extended to numerical differentiation problem in noisy environment. The obtained algebraic differentiators are non-asymptotic and robust against corrupting noises. Among these algebraic differentiators, the Jacobi differentiator has been used in many applications (see, e.g. [15-17]). In this paper, we summarize some existing error analysis results to give a strategy on how to chose the design parameters for the Jacobi differentiator. Then, we provide new algorithms which are more robust against the numerical errors produced by negative design parameters' values. Finally, we consider an application to nonlinear observation, where we compare the Jacobi differentiator to the high gain observer and the high order sliding modes differentiator.
\end{abstract}

Key Words: Algebraic differentiators, Noises error analysis, Nonlinear observation.

\section{Introduction}

The recent algebraic parametric method proposed by Fliess and Sira-Ramírez [1, 2] has been extended to numerical differentiation problem in noisy environment (see, e.g. [3-13]). The obtained so-called algebraic differentiators are divided into two classes: model-based differentiators and model-free differentiators. The formers were obtained by applying the algebraic method to a differential equation which defines a class of linear systems [3-5]. Hence, they were mainly used for linear systems. However, the model-free differentiators can be used for nonlinear systems. The first model-free differentiator was introduced in [6] by applying the algebraic method to the truncated Taylor series expansion of the signal to differentiate. Then, two model-free differentiators were studied in $[7,8]$, where the so-called Jacobi differentiator is the most used. Moreover, it was shown that the Jacobi differentiator can also be obtained by taking the truncated Jacobi orthogonal series expansion of the signal to differentiate. Then, it was significantly improved by admitting a time-delay $[7,8]$.

Recall that the Jacobi differentiator has some advantages. It is given by an integral formula in continuous case, which can be considered as a low-pass filter and corresponds to a convolution in discrete case. Thus, estimations at different instants can be obtained using a sliding integration window of finite length. Consequently, this differentiator is algebraic and non-asymptotic. Moreover, it exhibits good robustness properties with respect to corrupting noises (see [14] for more theoretical details). For these reasons, the Jacobi differentiator has been considered in a wide of real-time applications, such as nonlinear observation [15], fault diagnosis [16], model-free control [17], etc.

However, there was a lack of error analysis for the Jacobi differentiator. This differentiator depends on a set of design parameters, hence it is necessary to propose a strategy to chose these parameters so as to reduce estimation errors.
For this purpose, different errors for the Jacobi differentiator have been studied in [9-11], respectively. In particular, the definition domain of certain design parameters has been extended to negative values which can reduce the estimation errors, especially the time-delay. However, the proposed algorithms with negative design parameters' values were subject to numerical errors (see $[10,11]$ ).

The aim of this paper is given as follows. Firstly, by taking some existing error analysis results we summarize the design parameters' influence on each source of errors. Then, users of the Jacobi differentiator can chose these design parameters according to their need. Secondly, we provide new algorithms which are more robust against the numerical errors produced by negative design parameters' values. Thirdly, we compare the Jacobi differentiator to the high gain observer and the high order sliding modes differentiator by estimating the state variables of a nonlinear Ball and Beam system.

\section{Synthesis on Jacobi differentiator}

Consider a noise-corrupted measurement $x^{\varpi}: I \rightarrow \mathbb{R}$, $x^{\varpi}(t)=x(t)+\varpi(t)$, where $I$ is a finite time open interval of $\mathbb{R}^{+}, x \in \mathcal{C}^{n}(I)$ with $n \in \mathbb{N}$, and $\varpi$ is an additive corrupting noise. The objective is to estimate the $n^{\text {th }}$ order derivative of $x$ using $x^{\varpi}$. For this purpose, we apply a class of algebraic differentiators involving Jacobi polynomials, which were introduced in $[7,8]$ using a recent algebraic parametric method (see [13] for other algebraic differentiators).

In the next subsection, we show how to obtain these algebraic differentiators from the point of view of analysis, instead of algebra. First, for any $t_{0} \in I$, we introduce the set $D_{t_{0}}:=\left\{t \in \mathbb{R}_{+}^{*} ; t_{0}-t \in I\right\}$.

\subsection{Algebraic differentiators involving Jacobi polyno- mials}

For any $t_{0} \in I, x$ can be locally given on $\left[t_{0}-T, t_{0}\right]$ with $T \in D_{t_{0}}$ by the following Jacobi orthogonal series expan- 
sion:

$$
\begin{aligned}
& \forall \xi \in[0,1], x\left(t_{0}-T \xi\right)= \\
& \sum_{i \geq 0} \frac{\left\langle P_{i}^{(\mu, \kappa)}(\cdot), x\left(t_{0}-T \cdot\right)\right\rangle_{\mu, \kappa}}{\left\|P_{i}^{(\mu, \kappa)}\right\|_{\mu, \kappa}^{2}} P_{i}^{(\mu, \kappa)}(\xi),
\end{aligned}
$$

where $P_{i}^{(\mu, \kappa)}$ is the $i^{t h}$ order shifted Jacobi orthogonal polynomial defined on $[0,1]$ as follows (see [18] pp. 774-775):

$$
P_{i}^{(\mu, \kappa)}(\tau)=\sum_{j=0}^{i}\left(\begin{array}{c}
i+\mu \\
j
\end{array}\right)\left(\begin{array}{c}
i+\kappa \\
i-j
\end{array}\right)(\tau-1)^{i-j} \tau^{j},
$$

with $\mu, \kappa \in]-1,+\infty\left[,\langle\cdot, \cdot\rangle_{\mu, \kappa}\right.$ is a $\mathcal{L}^{2}([0,1])$ scalar product with the associated weight function $w_{\mu, \kappa}(\tau)=$ $(1-\tau)^{\mu} \tau^{\kappa}$, and the associated norm $\left\|P_{i}^{(\mu, \kappa)}\right\|_{\mu, \kappa}^{2}=$ $\frac{1}{2 i+\mu+\kappa+1} \frac{\Gamma(\mu+i+1) \Gamma(\kappa+i+1)}{\Gamma(\mu+\kappa+i+1) \Gamma(i+1)}$, where $\Gamma(\cdot)$ is the classical Gamma function (see [18] p. 255).

In order to approximate $x$ on $\left[t_{0}-T, t_{0}\right]$, we consider the classical polynomial approximation by taking the $N+1$ first terms in the Jacobi series expansion given in (1), and we denote the obtained $N^{t h}$ order polynomial by $D_{\kappa, \mu, T, N}^{(0)} x\left(t_{0}-\right.$ $T \cdot)$. Thus, we have:

$$
\begin{aligned}
& \forall \xi \in[0,1], D_{\kappa, \mu, T, N}^{(0)} x\left(t_{0}-T \xi\right):= \\
& \sum_{i=0}^{N} \frac{\left\langle P_{i}^{(\mu, \kappa)}(\cdot), x\left(t_{0}-T \cdot\right)\right\rangle_{\mu, \kappa}}{\left\|P_{i}^{(\mu, \kappa)}\right\|_{\mu, \kappa}^{2}} P_{i}^{(\mu, \kappa)}(\xi) .
\end{aligned}
$$

Hence, the $n^{\text {th }}$ order derivative of $x$ can be approximated by the one of $D_{\kappa, \mu, T, N}^{(0)} x\left(t_{0}-T \cdot\right)$. Inspired by this idea, the (causal) Jacobi differentiator is defined as follows [11]:

$$
\begin{aligned}
\forall \xi \in[0,1], D_{\kappa, \mu, T, q}^{(n)} x\left(t_{0}-T \xi\right):= \\
\quad \frac{1}{(-T)^{n}} \frac{d^{n}}{d \xi^{n}}\left\{D_{\kappa, \mu, T, N}^{(0)} x\left(t_{0}-T \xi\right)\right\},
\end{aligned}
$$

where $q=N-n \in \mathbb{N}$. It is shown in $[8,11]$ that this differentiator can also be obtained by taking the $q+1$ first terms in the Jacobi series expansion of $x^{(n)}$, i.e. we locally approximate $x^{(n)}$ by a $q^{t h}$ order polynomial on $\left[t_{0}-T, t_{0}\right]$ :

$$
\begin{aligned}
& \forall \xi \in[0,1], D_{\kappa, \mu, T, q}^{(n)} x\left(t_{0}-T \xi\right)= \\
& \sum_{i=0}^{q} \frac{\left\langle P_{i}^{(\mu+n, \kappa+n)}(\cdot), x^{(n)}\left(t_{0}-T \cdot\right)\right\rangle_{\mu+n, \kappa+n}}{\left\|P_{i}^{(\mu+n, \kappa+n)}\right\|_{\mu+n, \kappa+n}^{2}} P_{i}^{(\mu+n, \kappa+n)}(\xi) .
\end{aligned}
$$

Moreover, it can be given by the following integral formula:

$$
\begin{aligned}
\forall \xi \in[0,1], & D_{\kappa, \mu, T, q}^{(n)} x\left(t_{0}-T \xi\right)= \\
& \frac{1}{(-T)^{n}} \int_{0}^{1} Q_{\kappa, \mu, n, q, \xi}(\tau) x\left(t_{0}-T \xi\right) d \tau,
\end{aligned}
$$

where $C_{\kappa, \mu, n, i}=\frac{(\mu+\kappa+2 n+2 i+1) \Gamma(\kappa+\mu+2 n+i+1) \Gamma(n+i+1)}{\Gamma(\kappa+n+i+1) \Gamma(\mu+n+i+1)}$, $Q_{\kappa, \mu, n, q, \xi}(\tau)=w_{\mu, \kappa}(\tau) \sum_{i=0}^{q} C_{\kappa, \mu, n, i} P_{i}^{(\mu+n, \kappa+n)}(\xi) P_{n+i}^{(\mu, \kappa)}(\tau)$.
Finally, we substitute $x$ in (5) by $x^{\varpi}$ so as to obtain the Jacobi differentiator $D_{\kappa, \mu, T, q}^{(n)} x^{\varpi}\left(t_{0}-T \xi\right)$ in noisy case.

Different from the existing polynomial approaches, the idea of the Jacobi differentiator is to use a sliding integration window to estimate the value of $x^{(n)}$ at each $t_{0} \in I$ by $D_{\kappa, \mu, T, q}^{(n)} x\left(t_{0}-T \xi\right)$ with a fixed value of $\xi \in[0,1]$ (see $[7,8])$. If $\xi \neq 0$, then it produces a delay of value $T \xi$.

It is clear that for each $t_{0} \in I$, the Jacobi differentiator $D_{\kappa, \mu, T, q}^{(n)} x^{\varpi}\left(t_{0}-T \xi\right)$ depends on a set of design parameters, except for the order of the desired derivative $n$ :

- $\kappa, \mu \in]-1,+\infty[$ : the parameters of Jacobi polynomials,

- $q \in \mathbb{N}$ : the order of truncated Jacobi series expansion,

- $T \in D_{t_{0}}$ : the length of the sliding integration window,

- $\xi \in[0,1]$ : the parameter of time-delay $T \xi$.

In the next subsection, we give a general idea on how to chose these design parameters by giving some error analysis results.

\subsection{Error analysis in continuous case}

When we estimate $x^{(n)}\left(t_{0}\right)$ by $D_{\kappa, \mu, T, q}^{(n)} x\left(t_{0}-T \xi\right)$ with $\xi \in[0,1]$, the associated estimation error can be decomposed in continuous case as follows:

$$
\begin{gathered}
D_{\kappa, \mu, T, q}^{(n)} x^{\varpi}\left(t_{0}-T \xi\right)-x^{(n)}\left(t_{0}\right) \\
=\left(D_{\kappa, \mu, T, q}^{(n)} x^{\varpi}\left(t_{0}-T \xi\right)-D_{\kappa, \mu, T, q}^{(n)} x\left(t_{0}-T \xi\right)\right) \\
+\left(D_{\kappa, \mu, T, q}^{(n)} x\left(t_{0}-T \xi\right)-x^{(n)}\left(t_{0}-T \xi\right)\right) \\
\quad+\left(x^{(n)}\left(t_{0}-T \xi\right)-x^{(n)}\left(t_{0}\right)\right) .
\end{gathered}
$$

Hence, the Jacobi differentiator $D_{\kappa, \mu, T, q}^{(n)} x^{\varpi}\left(t_{0}-T \xi\right)$ is corrupted by two sources of errors:

- The noise error contribution:

$$
\begin{aligned}
& e_{\varpi}\left(t_{0} ; n, \kappa, \mu, T, q, \xi\right):= \\
& \quad D_{\kappa, \mu, T, q}^{(n)} x^{\varpi}\left(t_{0}-T \xi\right)-D_{\kappa, \mu, T, q}^{(n)} x\left(t_{0}-T \xi\right) .
\end{aligned}
$$

- The truncated term error due to the truncated Jacobi series expansion given in (4):

$$
\begin{aligned}
& e_{R}\left(t_{0} ; n, \kappa, \mu, T, q, \xi\right):= \\
& D_{\kappa, \mu, T, q}^{(n)} x\left(t_{0}-T \xi\right)-x^{(n)}\left(t_{0}\right) .
\end{aligned}
$$

Then, according to (6) the truncated term error can be divided into two parts:

- The amplitude error (in the vertical sense):

$$
\begin{aligned}
& e_{h}\left(t_{0} ; n, \kappa, \mu, T, q, \xi\right):= \\
& D_{\kappa, \mu, T, q}^{(n)}\left(t_{0}-T \xi\right)-x^{(n)}\left(t_{0}-T \xi\right) .
\end{aligned}
$$

$\circ$ The delay error due to $T \xi$ (in the horizontal sense):

$$
e_{v}\left(t_{0} ; n, \kappa, \mu, T, q, \xi\right):=x^{(n)}\left(t_{0}-T \xi\right)-x^{(n)}\left(t_{0}\right) .
$$

Consequently, each source of errors also depends on the design parameters. In order to show the influence of these parameters, we study the error bounds for these errors, which depend on the same parameters. For this purpose, we give a synthesis of some existing error analysis results.

1) Noise error contributions: In continuous case, we consider the noise errors due to the two following categories of noises. 
- Integrable noises: In this case the noise is assumed to be a bounded and integrable function on $I$, which can be divided into two parts [2]: the first part is a $(n-1)^{t h}$ order polynomial, considered as a structured perturbation, and the seconded part is a high frequency perturbation, considered as an unstructured noise.

An error bound based on the integral formula given in (5) was proposed in [13] (p. 90) for this kind of noise errors. Moreover, it was shown that the Jacobi differentiator $D_{\kappa, \mu, T, q}^{(n)} x^{\varpi}\left(t_{0}-T \xi\right)$ can eliminate a $(n-1)^{t h}$ order structured perturbation.

- Non-independent stochastic process noises: We consider in this case a class of continuous stochastic processes with finite second moments, whose mean value function and covariance kernel are continuous functions [19], such as Brownian motion and Poisson process.

Since a stochastic process usually is not bounded, the $\mathrm{Bi}$ enaymé-Chebyshev inequality was used to give error bounds by calculating the mean value and the variance of the associated noise error [10].

2) Amplitude error: Using the Taylor series expansion of $x$ at $t_{0}$, an error bound for the amplitude error was provided in [11], where the $(n+q+1)^{t h}$ order derivative of $x$ was assumed to be bounded on $I$.

3) Delay error: Instead of giving an error bound for the delay error, existing studies were based on how to chose $\xi$ and on how to reduce the delay $T \xi$ if $\xi \neq 0$.

- On the one hand, according to [8], we can take $\xi=\xi_{\kappa, \mu, q}^{(n)}$ in $D_{\kappa, \mu, T, q}^{(n)} x^{\varpi}\left(t_{0}-T \xi\right)$, where $\xi_{\kappa, \mu, q}^{(n)}$ is the smallest root of $P_{q+1}^{(\mu+n, \kappa+n)}$. This choice of $\xi$ significantly improved the Jacobi differentiator by admitting the time-delay $T \xi$. Indeed, it was shown in [8] that $e_{h}\left(t_{0} ; n, \kappa, \mu, T, q, \xi_{\kappa, \mu, q}^{(n)}\right)<$ $e_{h}\left(t_{0} ; n, \kappa, \mu, T, q+1,0\right)<e_{h}\left(t_{0} ; n, \kappa, \mu, T, q, 0\right)$. Moreover, the last inequality was confirmed in [11] using the amplitude error bound.

- On the other hand, $\xi_{\kappa, \mu, q}^{(n)}$ depends on three design parameters. The influence of $q$ is given by the classical orthogonal polynomial theory [20]. The influence of $\kappa$ et $\mu$ was studied in [10].

- Another choice of $\xi$ is to take $\xi=0.5$ (this case corresponds to the central Jacobi differentiator, see $[12,13])$. It is the optimal value of $\xi$ which minimizes the noise error contribution. However, the time-delay is equal to $0.5 T$. Hence, this choice of $\xi$ is only considered for off-line applications.

Finally, by numerically ${ }^{1}$ calculating the noise error bound, the amplitude error bound and the time-delay, we can know their behaviors with respect to different design parameters. Then, we can deduce the influence of these design parameters on each source of errors. We summarize the obtained results in Table 1 (see $[10,13]$ for more details), where the notations $a \uparrow, b \nearrow$ and $c \searrow$ mean that if we increase the value for the parameter $a$ then the error $b$ increases and the error $c$ decreases. Consequently, it is interesting to take negative values of $\kappa$ to reduce both the truncated term error and the noise error contribution (see [10] for more details). In the next subsection, we consider the errors in noisy discrete

\footnotetext{
${ }^{1}$ It is very difficult to analytically study the behavior of each error bound due to their complex expressions.
}

case.

\begin{tabular}{|c|c|c|c|}
\hline \hline & Stochastic noise error & Amplitude error & Time-delay \\
\hline$\kappa \uparrow$ & $\nearrow$ & $\nearrow$ & $\nearrow$ \\
\hline$\mu \uparrow$ & $\nearrow$ & $\searrow$ & $\searrow$ \\
\hline$q \uparrow$ & $\nearrow$ & $\searrow$ & $\searrow$ \\
\hline$T \uparrow$ & $\searrow$ & $\nearrow$ & $\nearrow$ \\
\hline \hline
\end{tabular}

Table 1: Influence of design parameters on $D_{\kappa, \mu, T, q}^{(n)} x\left(t_{0}\right.$ $\left.T \xi_{\kappa, \mu, q}^{(n)}\right)$ in continuous case.

\subsection{Error analysis in discrete case}

From now on, we assume that the noisy measurement $x^{\varpi}\left(t_{j}\right)=x\left(t_{j}\right)+\varpi\left(t_{j}\right)$ is given in discrete case with an equidistant sampling period $T_{s}$.

Since $x^{\varpi}$ is a discrete measurement, a numerical integration method is needed to approximate the integral formula given in (5). Let $\tau_{i}=\frac{i}{m}$ and $w_{i}>0$ for $i=0, \ldots, m$ with $m=\frac{T}{T_{s}} \in \mathbb{N}$ (except for $w_{0} \geq 0$ and $w_{m} \geq 0$ ) be respectively the abscissas and the weights for a given numerical integration method. Then, we get:

$$
\begin{array}{r}
D_{\kappa, \mu, T, q}^{(n)} x^{\varpi}\left(t_{0}-T \xi\right) \approx D_{\kappa, \mu, T, q}^{(n), m} x^{\varpi}\left(t_{0}-T \xi\right):= \\
\frac{1}{(-T)^{n}} \sum_{i=0}^{m} \frac{w_{i}}{m} Q_{\kappa, \mu, n, q, \xi}\left(\tau_{i}\right) x^{\varpi}\left(t_{0}-T \tau_{i}\right) .
\end{array}
$$

Consequently, the estimation error in this discrete noisy case is given by:

$$
\begin{aligned}
& D_{\kappa, \mu, T, q}^{(n), m} x^{\varpi}\left(t_{0}-T \xi\right)-x^{(n)}\left(t_{0}\right) \\
= & \left(D_{\kappa, \mu, T, q}^{(n), m} x^{\varpi}\left(t_{0}-T \xi\right)-D_{\kappa, \mu, T, q}^{(n), m} x\left(t_{0}-T \xi\right)\right) \\
& +\left(D_{\kappa, \mu, T, q}^{(n), m} x\left(t_{0}-T \xi\right)-D_{\kappa, \mu, T, q}^{(n)} x\left(t_{0}-T \xi\right)\right) \\
& \quad+\left(D_{\kappa, \mu, T, q}^{(n)} x\left(t_{0}-T \xi\right)-x^{(n)}\left(t_{0}\right)\right) \\
& e_{\varpi}^{m}\left(t_{0} ; n, \kappa, \mu, T, q, \xi\right)+e_{m}\left(t_{0}\right)+e_{R}\left(t_{0} ; n, \kappa, \mu, T, q, \xi\right),
\end{aligned}
$$

where $e_{\varpi}^{m}\left(t_{0} ; n, \kappa, \mu, T, q, \xi\right)$ is the discrete noise error contribution, $e_{m}\left(t_{0}\right)$ is the numerical error produced by a numerical integration method, and $e_{R}^{m}\left(t_{0} ; n, \kappa, \mu, T, q, \xi\right)$ is the truncated term error which is studied previously.

Remark that if $\kappa$ or $\mu$ is negative then the integral given in (5) is an improper integral due to $w_{\mu, \kappa}(\cdot)$. Hence, there can exist a singular value at $\tau=0$ or at $\tau=1$ when we apply a numerical integration method. In order to overcome this problem, the weight $w_{0}$ (resp. $w_{m}$ ) was set to zero in $[10,11]$ when $\kappa$ (resp. $\mu$ ) is negative. Based on this strategy, some error analysis was given.

\section{1) Discrete noise error contributions:}

Independent stochastic process noises: From now on, we consider a class of stochastic processes satisfying the following properties:

$\left(P_{1}\right)$ : for any $s, t \geq 0, s \neq t, \varpi(s)$ and $\varpi(t)$ are independent;

$\left(P_{2}\right)$ : the mean value function of $\{\varpi(t), t \in I\}$ belongs to $\mathcal{L}(I)$

$\left(P_{3}\right)$ : the variance function of $\{\varpi(t), t \in I\}$ is bounded on $I$. 
Note that white Gaussian noise and Poisson noise satisfy these conditions.

- The convergence in mean square of the discrete noisy error contributions with respect to the sampling period was studied in [10]. It was shown that the noise error contributions obtained with $\mu>-\frac{1}{2}$ and $\kappa>-\frac{1}{2}$ converge in mean square to zero when the sampling period tends to zero. Similar result was obtained in [14] using nonstandard analysis theory.

- For a set sampling period, by calculating the mean value and the variance of the associated noise errors the BienayméChebyshev inequality was used to give error bounds [11], so as to study the influence of the design parameters (the obtained results will be given in the next section).

\section{2) Numerical error:}

- When the values of $\kappa$ and $\mu$ are positive, we can take a small sampling period such that the numerical error can be negligible.

- When $\kappa$ or $\mu$ is negative, the numerical error is much larger than the one obtained with positive $\kappa$ and $\mu$. It can compensate the truncated term error such that the time-delay can be largely reduced in some cases (see $[10,11,13]$ ). However, these results are subject to the kind of signals to differentiate.

In the next section, we propose new algorithms for the Jacobi differentiator, which are more robust against the numerical error when the values of $\kappa$ and $\mu$ are negative.

\section{New algorithms for Jacobi differentiator}

\subsection{Change of variables}

It is shown in the previous section that if $\kappa$ (resp. $\mu$ ) is negative then there will be a singular value at $\tau=0$ (resp. $\tau=1$ ) in discrete case. In order to solve this problem, instead of taking $w_{0}=0$ (resp. $w_{m}=0$ ) in a numerical integration method, we apply a change of variables in (5) (see [21], p. 145).

- In the case where $-1<\kappa<0$ and $\mu \geq 0$ :

By taking the change of variables $\tau \rightarrow s^{\frac{1}{1+\kappa}}$, we get:

$$
\begin{aligned}
& D_{\kappa, \mu, T, q}^{(n)} x\left(t_{0}-T \xi\right)= \\
& \frac{1}{(-T)^{n}} \int_{0}^{1} Q_{\kappa, \mu, n, q, \xi}\left(s^{\frac{1}{1+\kappa}}\right) x\left(t_{0}-T s^{\frac{1}{1+\kappa}}\right) d s
\end{aligned}
$$

where

$$
\begin{aligned}
& Q_{\kappa, \mu, n, q, \xi}\left(s^{\frac{1}{1+\kappa}}\right)= \\
& \frac{\left(1-s^{\frac{1}{1+\kappa}}\right)^{\mu}}{1+\kappa} \sum_{i=0}^{q} C_{\kappa, \mu, n, i} P_{i}^{(\mu+n, \kappa+n)}(\xi) P_{n+i}^{(\mu, \kappa)}\left(s^{\frac{1}{1+\kappa}}\right) .
\end{aligned}
$$

- In the case where $-1<\mu<0$ and $\kappa \geq 0$ :

We first apply the change of variables $\tau \rightarrow 1-\tau$, we get:

$$
\begin{aligned}
& D_{\kappa, \mu, T, q}^{(n)} x\left(t_{0}-T \xi\right)=\frac{1}{(-T)^{n}} \times \\
& \int_{0}^{1} Q_{\kappa, \mu, n, q, \xi}(1-\tau) x\left(t_{0}-T(1-\tau)\right) d \tau .
\end{aligned}
$$

Then, similar to the first case, we take the change of variables $\tau \rightarrow s^{\frac{1}{1+\kappa}}$ in (14) to avoid the singular value at $\tau=0$.

- In the case where $-1<\mu<0$ and $-1<\kappa<0$ :
We first decompose the integral into two parts:

$$
\begin{aligned}
& D_{\kappa, \mu, T, q}^{(n)} x\left(t_{0}-T \xi\right)= \\
& \frac{1}{(-T)^{n}} \int_{0}^{\frac{1}{2}} Q_{\kappa, \mu, n, q, \xi}(\tau) x\left(t_{0}-T \tau\right) d \tau \\
& \quad+\frac{1}{(-T)^{n}} \int_{\frac{1}{2}}^{1} Q_{\kappa, \mu, n, q, \xi}(\tau) x\left(t_{0}-T \tau\right) d \tau .
\end{aligned}
$$

Then, we respectively apply the changes of variables proposed in the two previous cases to each integral obtained in (15) so as to avoid the singular values at $\tau=0$ and $\tau=1$.

In the following proposition, we give a new algorithm to implement the Jacobi differentiator with negative values of $\kappa$ in discrete noisy case.

Proposition 1 Let $x^{\varpi}\left(t_{j}\right)=x\left(t_{j}\right)+\varpi\left(t_{j}\right)$ for $j=$ $0, \ldots, M$, be a discrete noise-corrupted measurement given on a finite time open interval $I \subset \mathbb{R}^{+}$with an equidistant sampling period $T_{s}$, where $x \in \mathcal{C}^{n}(I)$ with $n \in \mathbb{N}$, and $\varpi$ is an additive corrupting noise. Then, for any $t_{j} \in I$ with $j=m, \ldots, M$, the $n^{\text {th }}$ order derivative of $x$ at $t_{j}$ is estimated by:

$$
\begin{aligned}
& D_{\kappa, \mu, m T_{s}, q}^{(n)} x^{\varpi}\left(t_{j}-m T_{s} \xi\right):= \\
& \quad \frac{1}{\left(-m T_{s}\right)^{n}} \sum_{i=0}^{m} \frac{w_{i}}{m^{1+\kappa}} Q_{\kappa, \mu, n, q, \xi}\left(\tau_{i}\right) x^{\varpi}\left(t_{j-i}\right),
\end{aligned}
$$

where $\tau_{i}=\frac{i}{m}, w_{i} \in \mathbb{R}_{+}$is the weight for a numerical integration method with numerical integration steps equal to $h_{i}=\frac{1}{m^{1+\kappa}}\left[(i+1)^{1+\kappa}-i^{1+\kappa}\right]$ for $i=0, \ldots, m-1$, and

$$
\begin{aligned}
& Q_{\kappa, \mu, n, q, \xi}\left(\tau_{i}\right)= \\
& \quad \frac{\left(1-\tau_{i}\right)^{\mu}}{1+\kappa} \sum_{i=0}^{q} C_{\kappa, \mu, n, i} P_{i}^{(\mu+n, \kappa+n)}(\xi) P_{n+i}^{(\mu, \kappa)}\left(\tau_{i}\right),
\end{aligned}
$$

$C_{\kappa, \mu, n, i}=\frac{(\mu+\kappa+2 n+2 i+1) \Gamma(\kappa+\mu+2 n+i+1) \Gamma(n+i+1)}{\Gamma(\kappa+n+i+1) \Gamma(\mu+n+i+1)}$, with $\kappa \in]-1,0\left[, \mu \in \mathbb{R}_{+}, M \geq m \in \mathbb{N}^{*}, q \in \mathbb{N}\right.$, and $\xi \in[0,1]$.

Proof. Since $x^{\varpi}$ is given in discrete case, we need to apply a numerical integration method to approximate the integral obtained in (13). Moreover, $x^{\varpi}$ is given with an equidistant sampling period, hence we need to take the abscissas $s_{i}$ for $i=0, \ldots, m$ with $m=\frac{T}{T_{s}}$, in such a way that $s_{i}^{\frac{1}{1+\kappa}}=\frac{i}{m}=$ $\tau_{i}$. Thus, the numerical integration steps become:

$$
h_{i}=s_{i+1}-s_{i}=\left(\frac{i+1}{m}\right)^{1+\kappa}-\left(\frac{i}{m}\right)^{1+\kappa},
$$

for $i=0, \ldots, m-1$. Finally, this proof is completed by considering the associated weights.

By applying a similar technique to (14) and (15) respectively, we can also obtain the algorithms of the Jacobi differentiator with negative values of $\mu$ in discrete noisy case.

\subsection{Error analysis in discrete case with new algorithms}

In this subsection, we use the new algorithms obtained in the previous subsection to give error analysis in discrete case for the Jacobi differentiator with negative values of $\kappa$ or $\mu$.

1) Numerical error: Since the singularity problem for the Jacobi differentiator with negative values of $\kappa$ or $\mu$ is solved 
in the previous subsection, we can take a small sampling period such that the numerical error can be negligible.

\section{2) Discrete noise error contributions}

- Non-independent stochastic process noises: We can take a small sampling period such that the discrete noise errors can be considered as the ones in continuous case. Hence, we consider that the results on the influence of design parameters obtained in continuous case are still available in discrete case for considered non-independent stochastic processes.

- Independent stochastic process noises: We focus on the class of stochastic processes satisfying the properties $\left(P_{1}\right)-\left(P_{3}\right)$ given in the previous section. In order to simplify notions, we denote the discrete noise errors $e_{\varpi}^{m}\left(t_{0} ; n, \kappa, \mu, T, q, \xi\right)$ produced with new algorithms by $e_{\varpi}^{m}$. Then, as done in $[9,10]$, sharp noise error bounds can be given using the Bienaymé-Chebyshev inequality: for any real number $\gamma>0$,

$$
M_{l}^{\gamma} \stackrel{p_{\gamma}}{<} e_{\varpi}^{m} \stackrel{p_{\gamma}}{<} M_{h}^{\gamma}
$$

where $M_{l}^{\gamma}=\mathbb{E}\left[e_{\varpi}^{m}\right]-\gamma \sqrt{\operatorname{Var}\left[e_{\varpi}^{m}\right]}, \quad M_{h}^{\gamma}=\mathbb{E}\left[e_{\varpi}^{m}\right]+$ $\gamma \sqrt{\operatorname{Var}\left[e_{\varpi}^{m}\right]}$, and $a \stackrel{p_{\gamma}}{<} b$ means that the probability for a real number $b$ to be larger than an other real number $a$ is equal to $p_{\gamma}$ with $p_{\gamma}>1-\frac{1}{\gamma^{2}}$. Since the considered noises are independent, it is easy to calculate $\mathbb{E}\left[e_{\varpi}^{m}\right]$ and $\operatorname{Var}\left[e_{\varpi}^{m}\right]$. Thus, we can study the design parameters' influence on the discrete noise error contributions $e_{\varpi}^{m}$ using the error bounds obtained in (17). In order to simplify this study, we assume that the noises also satisfy the following property:

$\left(P_{4}\right): \forall t_{j} \in I, \mathbb{E}\left[\varpi\left(t_{j}\right)\right]=0$ and $\operatorname{Var}\left[\varpi\left(t_{j}\right)\right]=1$.

Hence, we only need to study the variance of $e_{\varpi}^{m}$. Thus, by numerically calculating $\operatorname{Var}\left[e_{\varpi}^{m}\right]$, we can know its behavior with respect to different design parameters. Then, we can deduce these parameters' influence on $e_{\varpi}^{m}$. For instance, we show in Fig. 1 the behavior of $\operatorname{Var}\left[e_{\varpi}^{m}\right]$ with respect to $\kappa$ and $\mu$.

Finally, we summarize in Table 2 the results in discrete case obtained in the previous section and this section (see [13] for more details). In particular, we can see that for the proposed new algorithms, the optimal values of $\kappa$ and $\mu$ which minimize the noise error due to an independent noise are near to zero and positive.

\begin{tabular}{|c|c|c|}
\hline \hline & $\begin{array}{c}\text { Numerical error } \\
\text { with } w_{0} \text { or } w_{m}=0\end{array}$ & $\begin{array}{c}\text { Numerical error } \\
\text { with new algorithms }\end{array}$ \\
\hline$\kappa$ or $\mu<0$ & Large & Small \\
\hline \hline$\kappa \uparrow$ & $\begin{array}{c}\text { Independent Noise error } \\
\text { with } w_{0} \text { or } w_{m}=0\end{array}$ & $\begin{array}{c}\text { Independent Noise error } \\
\text { with new algorithm }\end{array}$ \\
\hline$\mu \uparrow$ & $\nearrow$ & $\searrow \nearrow\left(\right.$ op. at $\left.\approx 0_{+}\right)$ \\
\hline$q \uparrow$ & $\nearrow$ & $\searrow \nearrow$ (op. at $\left.\approx 0_{+}\right)$ \\
\hline$T \uparrow$ & $\nearrow$ & $\nearrow$ \\
\hline \hline
\end{tabular}

Table 2: Influence of design parameters on $D_{\kappa, \mu, T, q}^{(n)} x\left(t_{0}-\right.$ $\left.T \xi_{\kappa, \mu, q}^{(n)}\right)$ in discrete case.

\subsection{Strategy on choice of design parameters}

According to Table 1 and Table 2, the design parameters' influence on different errors is not the same, hence we should

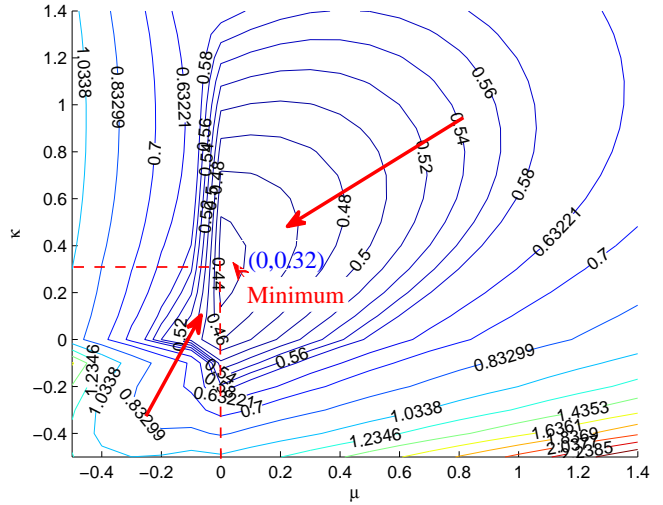

Fig. 1: $\operatorname{Var}\left[e_{\varpi}^{m}\right]$ with $n=1, T=1, q=1, \xi=\xi_{\kappa, \mu, 1}^{(1)}$ and $\kappa, \mu \in[-0.5,1.5]$.

take a compromise among these parameters. In this subsection, we give a general strategy on how to chose these parameters for the Jacobi differentiator.

1) Choice of $\xi$ : as shown previously [8], we take $\underline{\xi=\xi_{\kappa, \mu, q}^{(n)}}$, i.e. the smallest root of $P_{q+1}^{(\mu+n, \kappa+n)}(\cdot)$, in order to reduce the truncated term error by admitting a time-delay.

2) Choice of $q$ : According to Table 1 and Table 2, a large value of $q$ can produce a large noise error contribution, hence we should take a small value for $q$. As done in [7-11], we can take $q=0,1,2$. On the one hand, the truncated term error produced with $q=0$ is much larger than the one produced with $q=1$. This undesired effect is more important than the one that the noise error contribution produced with $q=1$ is larger than the noise error contribution produced with $q=$ 0 . On the other hand, the noise error contribution produced with $q \geq 2$ is much larger than the one produced with $q=1$. This undesired effect is more important than the one that the truncated term error produced with $q=1$ is larger than the one produced with $q \geq 2$. Indeed, thanks to the choice of $\xi$, the truncated term error produced with $q=1$ is significantly improved. Consequently, we propose to take $q=1$.

3) Choice of $(\kappa, \mu)$ : For the choices of $\mu$ and $\kappa$, we distinguish the two cases depending the nature of noises:

- Independent stochastic process noises: According to Table 2 , we take a positive value for $\mu$ which is near to 0 (smaller than 1) so as to avoid a large noise error contribution. Then, we chose the value of $\kappa$ according to the situation of applications. On the one hand, if one considers the noise effect is more important than the time-delay, then we can take a positive value of $\kappa$ and near to 0 (smaller than 1). On the other hand, if one prefers to produce a smaller time-delay, then we can take a negative value of $\kappa$.

- Non-independent stochastic process noises: According to Table 1, we take negative values for $\kappa$ and $\mu$ so as to reduce the noise error contribution. Meanwhile, a negative value of $\kappa$ can also reduce the truncated term error.

4) Choice of $T$ : The optimal value of $T$ which minimizes the amplitude error and the noise error contribution was proposed in [11]. However, this result was obtained using the bound of a higher order derivative of the considered signal and the bound of the noise, which are usually unknown in 
practical applications. Indeed, the choice of $T$ depends on the characteristic of the signal to differentiate and the variance of the noise. Hence, a criterion should be considered in order to choose an appropriate value of $T$ (see, e.g. [22]). This is beyond the scope of this paper.

5) Choice of numerical integration method: As mentioned previously, we can take a small sampling period such that the numerical error can be negligible. Then, a used numerical integration method only plays a role in the discrete noise error constructions, especially due to independent stochastic process noises. In order to show how to choose a numerical integration method, serval numerical integration methods, such as the trapezoidal rule, the rectangle rule, the Simpson's rule and the midpoint rule, have been compared in [13]. By considering a zero-mean white Gaussian noise, the associated discrete noise error constructions were obtained by applying each method respectively. Then, the variance values of these noise errors have been calculated and compared. According to the obtained results, the trapezoidal rule is proposed.

\section{Application to nonlinear observation}

According to a new approach of nonlinear observability based on differential algebra [23, 24], the numerical differentiation is an useful tool for nonlinear observation. A comparison between the Jacobi differentiator and the high order sliding modes differentiator has been done in [25]. In this section, we apply the Jacobi differentiator to the observation of a nonlinear Ball and Beam system. Moreover, we consider comparisons to two well-known methods.

\subsection{Ball and Beam system}

Ball and beam system is one of the most enduring popular and important laboratory models for teaching control systems engineering. Its dynamics can be described as follows:

$$
\left\{\begin{array}{cc}
\left(m r^{2}+J\right) \ddot{\theta}+2 m r \dot{r} \dot{\theta}+m g r \cos (\theta) & =u, \\
m \ddot{r}+m g \sin (\theta)-m r \dot{\theta}^{2} & =0,
\end{array}\right.
$$

where $m$ is the mass of the ball, $J$ is the length of the beam, $g$ is the gravitational constant, $r$ is the inertia position of the ball on the beam, $\theta$ is the beam angle and $u$ is the torque input. The state vector of this system is $(r, \dot{r}, \theta, \dot{\theta})^{T}$. Assume that the output vector is $\left(y_{1}, y_{2}\right)^{T}=(r, \theta)^{T}$, then we can verify that this system is observable using the algebraic approach [23, 24].

By taking $u=\left(m r^{2}+J\right) v+2 m r \dot{r} \dot{\theta}+m g r \cos (\theta)$ with $v \in \mathbb{R}$, the Ball and Beam system can be simplified. Then, the linearization around the beam angle $\theta=0$ gives us the following linear approximation of the system:

$$
\left\{\begin{array}{l}
\ddot{\theta}=v \\
\ddot{r}=-g \theta .
\end{array}\right.
$$

In order to stabilize the system, we take: $v=\frac{k_{3}}{g} r^{(3)}+$ $\frac{k_{2}}{g} r^{(2)}+\frac{k_{1}}{g} \dot{r}+\frac{k_{0}}{g} r$, where $k_{0}=w_{n_{1}}^{2} w_{n_{2}}^{2}, k_{1}=$ $2 \alpha\left(w_{n_{1}} w_{n_{2}}^{2}+w_{n_{2}} w_{n_{1}}^{2}\right), k_{2}=w_{n_{1}}^{2}+w_{n_{2}}^{2}+4 \alpha^{2} w_{n_{1}} w_{n_{2}}$ and $k_{3}=2 \alpha\left(w_{n_{1}}+w_{n_{2}}\right)$ with $\alpha=0.7, w_{n_{1}}=1 / \alpha$ and $w_{n_{2}}=3 w_{n_{1}}$.

By denoting the state vector by $z=\left(z_{1}, z_{2}, z_{3}, z_{4}\right)^{T}$, the system obtained in (19) can be divided into the two following sub-systems:

$$
\begin{aligned}
& \left\{\begin{array}{l}
\dot{R}=A R+B v, \\
y_{1}=r,
\end{array}\right. \\
& \left\{\begin{array}{l}
\dot{\Theta}=A \Theta+\Phi(\Theta, R), \\
y_{2}=\theta,
\end{array}\right.
\end{aligned}
$$

where $R=\left(\begin{array}{c}r \\ \dot{r}\end{array}\right), \Theta=\left(\begin{array}{c}\theta \\ \dot{\theta}\end{array}\right), A=\left(\begin{array}{cc}0 & 1 \\ 0 & 0\end{array}\right)$, $B=\left(\begin{array}{l}0 \\ 1\end{array}\right)$ and $\Phi(\Theta, R)=\left(\begin{array}{c}0 \\ -g \sin (\theta)-r \dot{\theta}^{2}\end{array}\right)$.

In the next subsection, we estimate $(r, \dot{r}, \theta, \dot{\theta})^{T}$ from the noisy output $y_{1}^{\varpi}$ and $y_{2}^{\varpi}$.

\subsection{Comparisons to two existing methods}

\subsubsection{High gain observer}

During the past few years, the high gain observer played an important role in the design of nonlinear output feedback control of nonlinear systems [26, 27]. It is mainly used to estimate the derivatives of the output.

By applying the high gain observer to (20) and (21) respectively, we get:

$$
\begin{aligned}
& \left\{\begin{array}{l}
\dot{\widehat{R}}=A \widehat{R}-K_{r}\left(\widehat{y}_{1}-y_{1}\right)+B v, \\
\widehat{y}_{1}=\hat{r},
\end{array}\right. \\
& \left\{\begin{array}{l}
\dot{\widehat{\Theta}}=A \widehat{\Theta}-K_{\theta}\left(\widehat{y}_{2}-y_{2}\right)+\Phi(\widehat{\Theta}, \widehat{R}), \\
\widehat{y}_{2}=\hat{\theta},
\end{array}\right.
\end{aligned}
$$

where $\widehat{R}=\left(\begin{array}{c}\hat{r} \\ \hat{\dot{r}}\end{array}\right)$ are $\widehat{\Theta}=\left(\begin{array}{c}\hat{\theta} \\ \hat{\dot{\theta}}\end{array}\right)$ present the estimated state variables. The gains are given by $K_{r}=S_{\infty}^{-1}\left(\alpha_{r}\right) C$ and $K_{\theta}=S_{\infty}^{-1}\left(\alpha_{\theta}\right) C$, where $S_{\infty}^{-1}(\alpha)$ is the unique solution of the matrix equation:

$$
\left\{\begin{aligned}
\alpha S_{\infty}(\alpha)+A^{T} S_{\infty}(\alpha)+S_{\infty}(\alpha) A & =C C^{T} \\
S_{\infty}(\alpha) & =S_{\infty}^{T}(\alpha)
\end{aligned}\right.
$$

where $C=\left(\begin{array}{l}1 \\ 0\end{array}\right)$. Consequently, we get $K_{r}=\left(\begin{array}{c}2 \alpha_{r} \\ \alpha_{r}^{2}\end{array}\right)$ and $K_{\theta}=\left(\begin{array}{c}2 \alpha_{\theta} \\ \alpha_{\theta}^{2}\end{array}\right)$ with $\alpha_{r} \in \mathbb{R}$ and $\alpha_{\theta} \in \mathbb{R}$.

\subsubsection{High order sliding modes differentiator}

The high order sliding modes differentiator described in [28] can be expressed in a dynamic form as follows:

$\left\{\begin{array}{l}\dot{\alpha}_{0}=-\lambda_{n} W^{\frac{1}{n+1}}\left|\alpha_{0}-x\right|^{\frac{n}{n+1}} \operatorname{sign}\left(\alpha_{0}-x\right)+\alpha_{1}, \\ \dot{\alpha}_{1}=-\lambda_{n-1} W^{\frac{1}{n}}\left|\alpha_{1}-\dot{\alpha}_{0}\right|^{\frac{n-1}{n}} \operatorname{sign}\left(\alpha_{1}-\dot{\alpha}_{0}\right)+\alpha_{2}, \\ \vdots \\ \dot{\alpha}_{n-1}=-\lambda_{1} W^{\frac{1}{2}}\left|\alpha_{n-1}-\dot{\alpha}_{n-2}\right|^{\frac{1}{2}} \operatorname{sign}\left(\alpha_{n-1}-\dot{\alpha}_{n-2}\right)+\alpha_{n}, \\ \dot{\alpha}_{n}=-\lambda_{0} W \operatorname{sign}\left(\alpha_{n}-\dot{\alpha}_{n-1}\right),\end{array}\right.$

where $x \in \mathcal{C}^{n+1}$ is the signal to differentiate, and $W$ is a constant such that $\left\|x^{(n+1)}\right\|_{\infty} \leq W$. It was shown in [28] that, if the gains $\lambda_{i}$ for $i=0,1, \ldots, n$, are chosen properly, then the differentiator converges in a finite time $T$. For $n=$ 5 , the gains can be chosen as $\lambda_{0}=1.1, \lambda_{1}=1.5, \lambda_{2}=3$, $\lambda_{3}=5, \lambda_{4}=8$ and $\lambda_{5}=12$. In order to estimate $r$ and $\dot{r}$ (resp. $\theta$ and $\dot{\theta}$ ), we apply this differentiator to (20) (resp. (21)) with $n=2$ and $x=y_{1}$ (resp. $x=y_{2}$ ). 


\subsubsection{Simulation results}

We first consider the high gain observer and the sliding modes differentiator. On the one hand, when using the high gain observer for the Ball and Beam system, there are two design parameters to set: $\alpha_{r}$ and $\alpha_{\theta}$. During the implementations of the high gain observer, we observe that it is necessary to take large values for these parameters to reduce the estimation errors in noise-free case. However, large values of these parameters can produce large noise errors in noisy case. Hence, there is also a compromise to consider. In the following simulations, we take $\alpha_{r}=10$ and $\alpha_{\theta}=30$ so as to produce small estimation errors in noise-free case.

On the other hand, by simulating the Ball and Beam system we obtain that $r^{(2)} \leq 2$ and $\theta^{(2)} \leq 6.7$. Hence, we respectively take $W_{r}=2$ and $W_{\theta}=6.7$ for the sliding modes differentiator. However, these values are usually unknown in practice applications. Moreover, during the implementations of this differentiator in noise-free case, we observe that it is not robust against a "large" sampling period, especially when it is used to estimate $\dot{r}$ and $\dot{\theta}$ (see [13] for more details). In order to solve this problem, we assume $y_{1}$ and $y_{2}$ are measured with an equidistant sampling period $T_{s}=0.0001 \mathrm{~s}$ in the following simulations. Moreover, we assume that $y_{1}^{\varpi}$ (resp. $y_{2}^{\varpi)}$ is the discrete output corrupted by a noise $\varpi$ which is a zero-mean Gaussian iid sequence and whose variance is adjusted in such a way that $\mathrm{SNR}=24.5 \mathrm{~dB}$ (resp. $\mathrm{SNR}=23.6 \mathrm{~dB}$ ).

For the implementation of the Jacobi differentiator, we need four sets of design parameters for each state variable. According to the previous study on the choice of the design parameters, we take $q=1$ and $\mu=0$. For the value of $\kappa$, we consider two cases:

- Firstly, according to Fig. 1 we take $\kappa_{1}=0.32$ to reduce the noise error contribution.

- Secondly, we take $\kappa_{2}=-0.5$ to reduce the time-delay and the amplitude error.

Then, we take $\xi=\xi_{\kappa_{i}, 0,1}^{(n)}$ for $n=1,2, i=1,2$, in the two cases respectively. Finally, the only parameter to set is the length of each sliding window. However, we need four different values of $T$ to estimate each state.

Finally, we give the obtained estimations of $\dot{\theta}$ in Fig. 2 and Fig. 3. Since the values of the time-delay produced by the Jacobi differentiator can be calculated, we can see that by shifting the corresponding estimations we get smaller estimation errors than the other two methods. Moreover, when the value of $\kappa$ is negative, the time-delay is reduced, however the noise error contribution is increased. According to the simulations, we summarize the comparison results in Table 3 by considering other different criteria.

\begin{tabular}{|c|c|c|}
\hline \hline Method & Convergence time & Number of parameters \\
\hline Jacobi & $T$ & Number of state variables: 4 \\
\hline High gain & unknown & Number of sub-systems: 2 \\
\hline Sliding modes & unknown & Number of sub-systems: 2 \\
\hline \hline Method & Time-delay & Robustness to noise and $T_{s}$ \\
\hline Jacobi & known $\left(T \xi_{\kappa, 0,2}^{(n)}\right)$ & good \\
\hline High gain & unknown $($ small $)$ & good \\
\hline Sliding modes & unknown & bad \\
\hline \hline
\end{tabular}

Table 3: Comparison results with different criteria

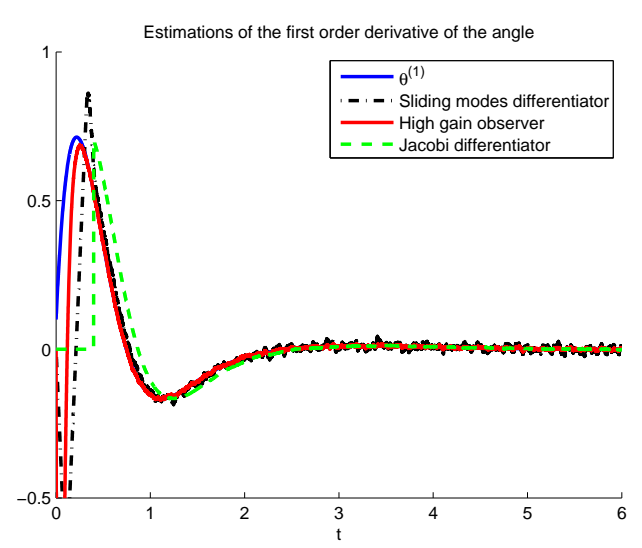

(a) Estimations for $\dot{\theta}$

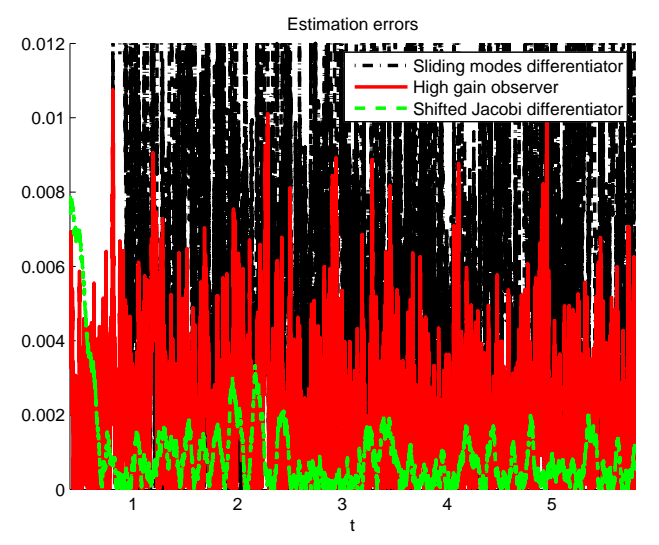

(b) Absolute estimation errors.

Fig. 2: $D_{\kappa, \mu, T, q}^{(n), m} x\left(t_{0}-T \xi_{\kappa, \mu, q}^{(n)}\right)$ with $n=1, \underline{\kappa=0.32}, \mu=$ $0, q=1, \underline{\xi_{\kappa, \mu, q}^{(n)}=0.3088}$ and $T=0.4$.

\section{Conclusions}

In this paper, first we have shown how to obtain the socalled algebraic Jacobi differentiator from the point of view of analysis. Then, we have summarized some existing error analysis results for the Jacobi differentiator. A synthesis of the design parameters' influence on each source of errors has been provided in continuous case, where we recalled that negative values of $\kappa$ can reduce both the truncated term error and the noise error contributions. Error analysis in discrete case has also been recalled, where the existing algorithm was not robust against the numerical errors obtained with negative values of $\kappa$. Hence, new algorithms have been proposed to overcome this issue. Based on these new algorithms, error analysis has been given in discrete case. Then, a strategy on how to chose the design parameters has been proposed. The obtained parameters' influence on the errors was deduced from the one on the corresponding error bounds. In general, it is impossible to find a set of optimal parameters which are suitable for all situations. Users should take parameters according to their need. For instance, we can take negative values of $\kappa$ (resp. positive values of $\kappa$ and smaller than 1) to reduce the time-delay (resp. to reduce the noise effect due to an independent noise). The largest difficulty is to chose the parameter $T$ (the length of the sliding integration window), which depends on the nature of the signal to differentiate and the one of noises. Hence, as shown in [22], a criterion should 


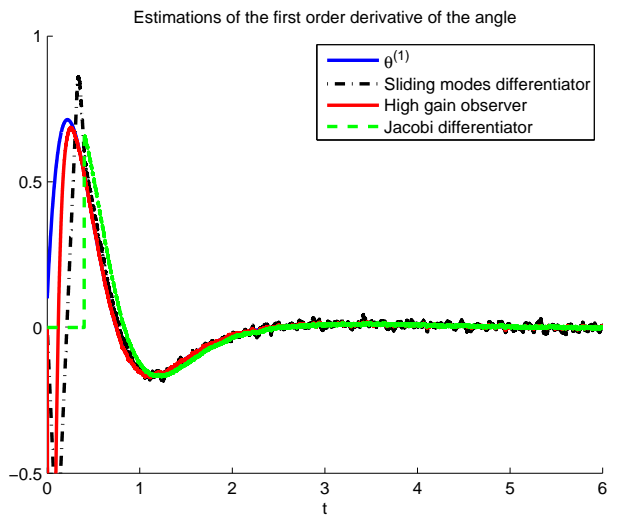

(a) Estimations for $\dot{\theta}$.

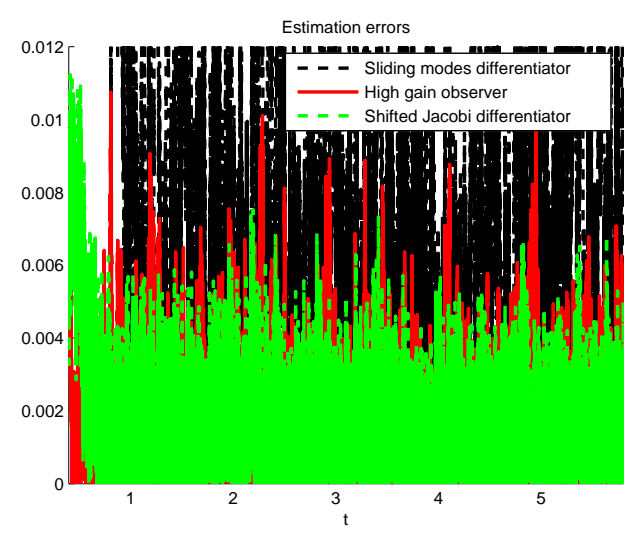

(b) Absolute estimation errors.

Fig. 3: $D_{\kappa, \mu, T, q}^{(n), m} x\left(t_{0}-T \xi_{\kappa, \mu, q}^{(n)}\right)$ with $n=1, \underline{\kappa=-0.5}, \mu=$ $0, q=1, \underline{\xi_{\kappa, \mu, q}^{(n)}=0.2198}$ and $T=0.4$.

be considered in order to choose an appropriate value of $T$. This is beyond the scope of this paper. Finally, the Jacobi differentiator has been compared to the high gain observer and the high order sliding modes differentiator by considering different criteria. It has been shown that the Jacobi differentiator can give smaller estimation errors by admitting a time-delay.

\section{References}

[1] M. Fliess and H. Sira-Ramrez, An algebraic framework for linear identification, ESAIM Control Optim. Calc. Variat., 9: 151168, 2003.

[2] M. Fliess, M. Mboup, H. Mounier and H. Sira-Ramírez, Questioning some paradigms of signal processing via concrete examples, in Algebraic Methods in Flatness, Signal Processing and State Estimation, 1-21, G. Silva-Navarro and H. SiraRamírez, editeurs, Editiorial Lagares, 2003.

[3] M. Fliess and H. Sira-Ramrez, Reconstructeurs d'états, Comptes Rendus Mathematique, 338(1): 91-96, 2004.

[4] Y. Tian, T. Floquet and W. Perruquetti, Fast state estimation in linear time-varying systems: an algebraic approach, in Proc. 47th IEEE Conference on Decision and Control, Cancun Mexique, 2008.

[5] Y. Tian, Une contribution à l'observation et à l'estimation des systèmes linéaires, INRIA Lille Nord Europe, Ecole Centrale de Lille, Phd thesis in French, defense on December 8, 2010.

[6] M. Fliess, C. Join, M. Mboup and H. Sira-Ramírez, Compression différentielle de transitoires bruités, Comptes Rendus
Mathematique, 339(11): 821-826, 2004

[7] M. Mboup, C. Join and M. Fliess, A revised look at numerical differentiation with an application to nonlinear feedback control, in Proc. 15th Mediterranean conference on Control and automation (MED'07), Athenes, Greece, 2007.

[8] M. Mboup, C. Join and M. Fliess, Numerical differentiation with annihilators in noisy environment, Numerical Algorithms, 50(4): 439-467, 2009.

[9] D.Y. Liu, O. Gibaru and W. Perruquetti, Error analysis for a class of numerical differentiator: application to state observation, in Proc. 48th IEEE Conference on Decision and Control, Shanghai, China, 2009.

[10] D.Y. Liu, O. Gibaru and W. Perruquetti, Error analysis of Jacobi derivative estimators for noisy signals, Numerical Algorithms, 58(1): 53-83, 2011.

[11] D.Y. Liu, O. Gibaru and W. Perruquetti, Convergence Rate of the Causal Jacobi Derivative Estimator, Curves and Surfaces 2011, LNCS 6920 proceedings, 45-55, 2011.

[12] D.Y. Liu, O. Gibaru and W. Perruquetti, Differentiation by integration with Jacobi polynomials, Journal of Computational and Applied Mathematics, 235(9): 3015-3032, 2011.

[13] D.Y. Liu, Error analysis of a class of derivative estimators for noisy signals and applications, INRIA Lille Nord Europe \& Universit Lille 1, Ph.D. thesis, defense on October 17, 2011. Available at http://tel.archives-ouvertes.fr/tel-00634652/fr/.

[14] M. Fliess, Analyse non standard du bruit, Comptes Rendus Mathematique, 342(10): 797-802, 2006.

[15] L. Yu, J.P. Barbot, D. Boutat and D. Benmerzouk, Observability forms for switched systems with zeno phenomenon or high switching frequency, IEEE Trans. on Automatic Control, 56(2): 436-441, 2011.

[16] L. Kiltz, M. Mboup and J. Rudolph, Fault diagnosis on a magnetically supported plate, in Proc. 1st Int. Conf. on Systems and Computer Science, Villeneuve dAscq, France, 2012.

[17] M. Fliess and C. Join, Commande sans modèle et commande à modèle restreint, $e$-STA, 5(4): 1-23, 2008.

[18] M. Abramowitz and I.A. Stegun, editeurs, Handbook of mathematical functions, GPO, 1965.

[19] E. Parzen, Stochastic processes, Holden-Day, San Francisco, 1962.

[20] G. Szegö, Orthogonal polynomials, 3rd edn. AMS, Providence, RI 1967.

[21] W.H. Press, S.A. Teukolsky, W.T. Vetterling and B.P. Flannery, Numerical Recipes in C, Second Edition, Cambridge, UK: Cambridge University Press, 1992.

[22] L. Kiltz and J. Rudolph, Parametrization of algebraic numerical differentiators to achieve desired filter characteristics, in Proc. 52nd IEEE Conference on Decision and Control, Florence, Italy. 2013.

[23] M. Fliess and S. Diop, Nonlinear observability, identifiability and persistent trajectories, in Proc. 36th IEEE Conference on Decision and Control, 1991.

[24] S. Diop and M. Fliess, On nonlinear observability, in Proc. 1st European Control Conference, Grenoble, 1991.

[25] L. Sidhom, Sur les différentiateurs en temps réel: algorithmes et applications, INSA de Lyon, Ph.D. thesis, defense on September 29, 2011.

[26] J.P. Gauthier, H. Hammouri and S. Othman, A simple observer for nonlinear systems applications to bioreactors, IEEE Transactions on Automatic Control, 37(6): 875-880, 1992.

[27] F. Deza, E. Busvelle, J.P. Gauthier and D. Rakotopara, High gain estimation for nonlinear systems, Systems \& control letters, 18(4): 295-299, 1992.

[28] A. Levant, Higher-order sliding modes, differentiation and output-feedback control, International Journal of Control, 76 : 924-941, 2003. 